\title{
¿Se puede hablar de un arte queer español?
}

\author{
Juan Jesús Montiel Rozas \\ Universidad de Málaga \\ juanmontielhistoriadelarte@gmail.com
}

RESUMEN: Hasta la llegada de la década de los noventa la producción artística y teórica en España relativa a las cuestiones de género era prácticamente inexistente. No obstante, a partir de dicha década el feminismo comienza a desarrollarse y difundirse dentro de las fronteras nacionales a la par que la teoría queer da sus primeros pasos. En poco más de veinte años estos estudios han avanzado de un modo vertiginoso consiguiendo erigir, ya en el 2000, a algunos teóricos y artistas que son reconocidos a nivel internacional. En este artículo se reflexiona sobre una de las consecuencias derivadas de la rapidez con que se ha producido el proceso: si tiene sentido hablar de un arte queer español.

PALABRAS CLAVE: Arte contemporáneo, España, Activismo, Queer, Feminismo, Estudios de género.

\section{Can we talk about a Spanish Queer Art?}

ABSTRACT: Until the 1990s, the Spanish artistic and theoretical gender-related production was almost nonexistent. However, it is from that decade on when feminism begins to develop and spread within the borders of the country, and it is also at this time when the Queer theory takes its firsts steps. These studies have developed in such a fast way in just over 20 years, that, already in 2000 , we can find some theorists and artists who are recognized in the international context. This paper reflects on one of the consequences resulting of the celerity of this process and thus whether or not it makes sense to talk about a Spanish Queer Art.

KEY WORDS: Contemporary Art, Spain, Activism, Queer Art, Feminism, Gender Studies.

Recibido: 6 de marzo de 2015 / Aceptado: 7 de octubre de 2015.

En 1997 Juan Vicente Aliaga, uno de los teóricos españoles más reputados en el campo de los estudios de género, publicó un artículo titulado: “¿Existe un arte queer en España?»`. En este texto, capital para las investigaciones relativas a las relaciones entre arte y género en España, explicó tanto la breve historia como lo resbaladizo del concepto queer. Tras ello comentaba la obra de colectivos y artistas como LSD, Jesús Martínez Oliva o Roberto González Fernández en cuya producción el cuestionamiento de los roles tradicionales de género era una constante. Sin embargo, no respondió a la pregunta del título. Por un lado, se puede hablar de una producción artística dentro del ámbito español relativa a las problemáticas del cuerpo y a la identidad sexual, eso es indudable. Por otro, no obstante, ¿se le puede aplicar el término anglosajón queer?

Para responder a esa pregunta, al igual que en este texto, lo mejor sería comenzar explicando qué es el término queer y en qué momento y contexto se produjo su importación a España. En 1969 Nueva York fue el escenario de las denominadas revueltas de Stonewall. Estas marcaron el inicio del movimiento homosexual norteamericano. Esto, junto a la fuerte ola de activismo que se desarrolló posteriormente y que se expandió durante la década de los ochenta con motivo de la reacción por parte de los gobiernos ante la crisis del sida, convertirá a Estados Unidos en el punto de referencia en lo relativo a los movimientos de liberación homosexual. Fue en este contexto contestatario en el que se subvirtió el empleo del insulto queer,

MONTIEL ROZAS, Juan Jesús: “¿Se puede hablar de un arte queer español?», Boletín de Arte, n. ${ }^{\circ}$ 36, Departamento de Historia del Arte, Universidad de Málaga, 2015, pp. 103-113, ISSN: 0211-8483. 
que podría traducirse como raro o como un modo de referirse de un modo despectivo a los gais, lesbianas, bisexuales, travestis y transexuales, para pasar a ser un medio de autoafirmación². A finales de los ochenta y en los noventa pasará a ser una teoría y adquirir un papel predominante dentro de los actuales estudios de género.

Obviamente, el ámbito artístico no fue ajeno a esta teoría y así como el feminismo y los Men's Studies habían tenido un reflejo en él, lo queer también lo hará, tal y como demuestra la obra de De LaGrace Volcano o Catherine Opie, por ejemplo. No obstante, desde mi punto de vista, aquello que determina al Queer Art, aunque supone un contrasentido puesto que la teoría queer es contraria a las etiquetas, es la indefinición. Son obras que representan estados intermedios. No hablan de un género, una identidad o una orientación sexual. Si aceptamos esta premisa podemos remontarnos a las primeras décadas del siglo XX para encontrar obras que, en Europa, hagan lo propio como Rrose Sélavy de Marcel Duchamp de 1920 o la producción de Claude Cahun, Hannah Höch y Pierre Molinier [1]. Por ello, podría afirmarse que el desarrollo y florecimiento de los Queer Studies en los noventa más que introducir un nuevo tema dentro del mundo del arte, lo que hizo fue iniciar un proceso de popularización que llega hasta nuestros días.

Ahora bien, ¿y en España? ¿Cuándo se genera este movimiento? En primer lugar hay que destacar que en España este proceso se produjo de manera tardía, de hecho, se inició en los noventa con escaso éxito para, en el 2000, ser una fuente de producción teórica muy prolífica.

Esta particularidad se vio motivada, principalmente, por la represión llevada a cabo durante el franquismo hacia todo aquello que tuviera que ver con la homosexualidad ya no solo por el Estado, sino por los propios ciudadanos a través de acusaciones, detenciones y redadas ${ }^{3}$. Dentro de este ambiente represivo, desde una perspectiva jurídica la situación del homosexual se recrudece a través de una enmienda y una nueva ley. La enmienda, realizada en 1954, consistió en un endurecimiento de la ley de vagos y maleantes (1933), que fue creada con el objetivo de eliminar del espacio público a aquellos sujetos inmorales o peligrosos tales como drogadictos, mendigos o estafadores ${ }^{4}$. A pesar de que la homosexualidad no era nombrada expresamente en ella, quedaba enmarcada dentro de su campo de acción. Fue con la enmienda de 1954 con la que se soluciona dicha ambigüedad categorizando al homosexual como un sujeto susceptible de ser procesado por el aparato jurídico debido a su condición sexual ${ }^{5}$. Sin embargo, en 1970 esta se vio reemplazada con la promulgación de la Ley de Peligrosidad y Rehabilitación Social (LPRS) ${ }^{6}$. A través de ella se corrigieron las ambigüedades de la anterior castigando ya no solo la homosexualidad, sino cualquier tipo de acto relacionado entre los cuales podría entenderse el afeminamiento ${ }^{7}$.

Otro de los agentes determinantes fue la Transición Democrática en la segunda mitad de los setenta, momento en el que, siendo precedidos por la fundación de la asociación Movimiento Español de Liberación Homosexual (MEHL) en 1972 en Barcelona, comienzan a surgir otros grupos activistas en distintas ciudades de España, y en 1977 tiene lugar la primera manifestación del Día del OrguIlo Gai dentro del territorio español ${ }^{8}$. Sin embargo, pese a que la comunidad LGTB pudo disfrutar de una mayor visibilidad gracias a los distintos movimientos contraculturales que surgen en ciudades como Madrid o Barcelona, la LPRS no deja de utilizarse hasta 1978, año en el que se retira la homosexualidad de esta ley y pasa a llamarse ley de escándalo público. Aún con este contexto favorable, como argumentan Jorge Luis Marzo y Guillem Martínez, durante la Transición se persiguió la idea de crear una idea única y consensuada de España lo que, a su vez, suponía el evitar cualquier discurso o movimiento social que supusiera una fragmentación de los valores que el Estado defendía ${ }^{9}$. Entre ellos, obviamente, se situaban los movimientos activistas y teóricos feministas y LGTB.

A ambos motivos habría que añadir el hecho de que España carecía de una producción teórica y artística feminista que, como en otros casos como el de Estados Unidos y Reino Unido, antecediera a las prácticas artísticas y discursivas queer. Según Patricia Mayayo los motivos de esta situación serían:

En primer lugar, la renuencia de las propias artistas experimentales de los setenta a reconocerse como "feministas". [...], la estigmatización del término feminista en una sociedad tan machista como era la del tardofranquismo [...] y la prioridad que tenía en España la lucha contra la dictadura frente a cualquier otro tipo de reivindicación política hicieron que muchas artistas manifestasen reticencias con respecto al movimiento de mujeres ${ }^{10}$. 
Así, la persecución que recibió la homosexualidad hasta mediados de los ochenta junto con la despolitización de los discursos artísticos fruto de las políticas culturales de la Transición determinó una producción artística oficial muy característica durante los setenta y ochenta. En ella, como dice Juan Vicente Aliaga en el citado texto al inicio de este artículo, "cuando de retratar el cuerpo se trataba lo hacían relegando su dimensión sexuada/política, incidiendo por el contrario en aspectos intrascendentes» ${ }^{11}$.

Sin embargo, podría trazarse un discurso paralelo partiendo de la ciudad de Barcelona, fuertemente marcada por la ideología nacionalista puesto que lo que unos años más tarde pasarían a ser los primeros grupos activistas LGTB del país encontraron en dicha ideología una aliada en contra de la opresión dictatorial a pesar de que, posteriormente, siguieron caminos divergentes. Como dice Alberto Mira, «el nacionalismo proporcionaba herramientas retóricas de gran importancia: el orgullo de pertenecer a una minoría, la justificación de la lucha contra la opresión (sea heterosexista o españolista) y, sobre todo, la imagen de un horizonte utópico por el que merecía la pena luchar $»^{12}$.

Entre ambos casos destaca el de Barcelona por ser la primera ciudad en acoger a una organización homófila, a un grupo activista LGTB y la primera manifestación del Día del Orgullo Gai'13. Los motivos que posibilitaron dicha situación son el hecho de ser una ciudad portuaria, ser uno de los principales destinos del turismo internacional en España y que en la década de los setenta fuera un puente para todos aquellos que viajaban a Ibiza ${ }^{14}$. Dentro de este último conjunto habría que destacar a los norteamericanos, quienes portaban con ellos una cultura en la que las asociaciones homófilas habían precedido en varios años a la aparición de los grupos activistas LGTB ${ }^{15}$. Ahora bien, si en Estados Unidos hubo una clara falta de consenso entre homófilos y activistas, en España y, más concretamente, en Barcelona, en cambio, la Agrupación Homófila para la Igualdad de Sexos (AGHOIS) paso a ser en 1972 MEHL, es decir, un grupo activista $^{16}$.

Dentro de su entramado urbano, si l'Eixample fue la zona donde residió la burguesía, El Raval, las Ramblas y la Plaza Real, debido a su cercanía al puerto, concentraron a todo aquel conjunto de personas repudiadas por la sociedad normativa: prostitutas, delincuentes, homosexuales, pícaros, etc. Todos ellos formaban un microcosmos en el que el

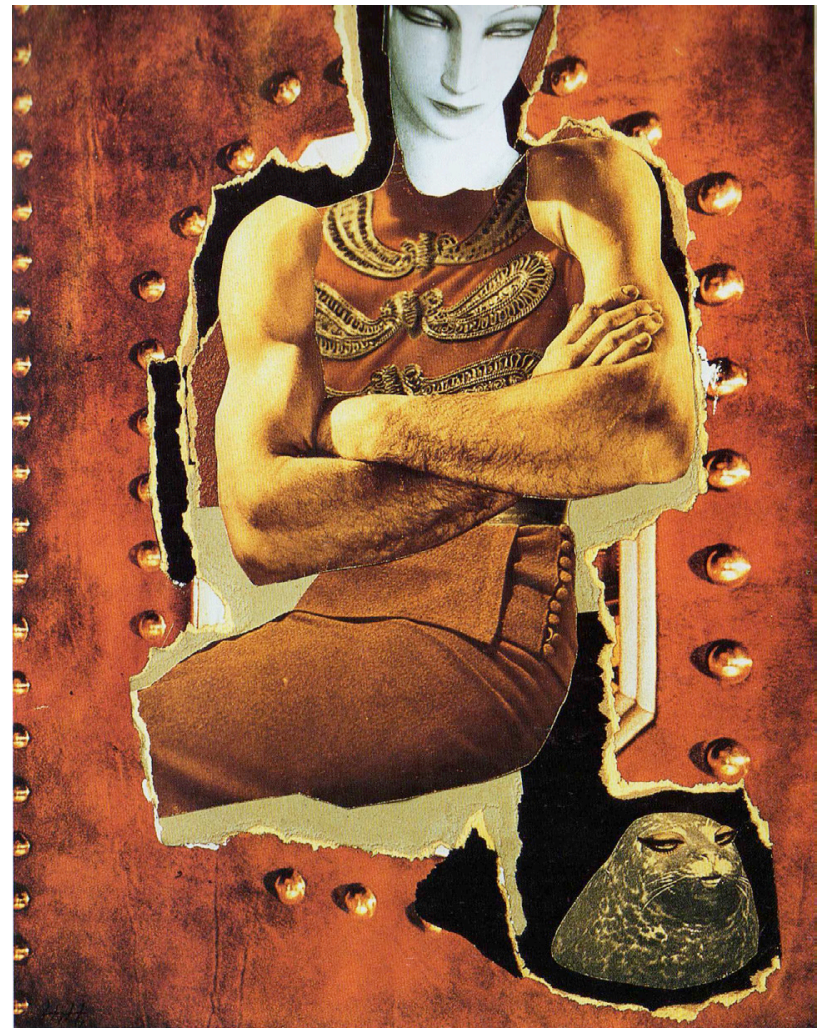

1. Hannah Höch, Domadora, 1930

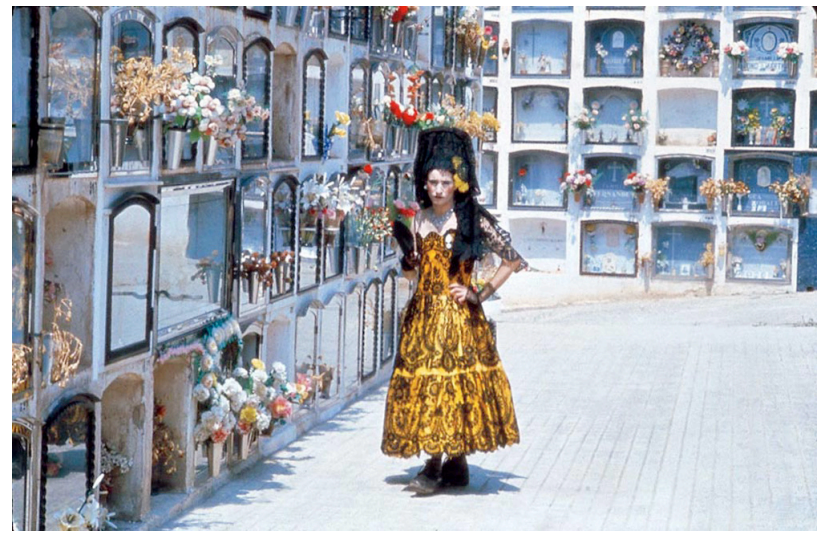

2. Fotograma de la película de 1978 Ocaña, retrat intermitent de Ventura Pons 


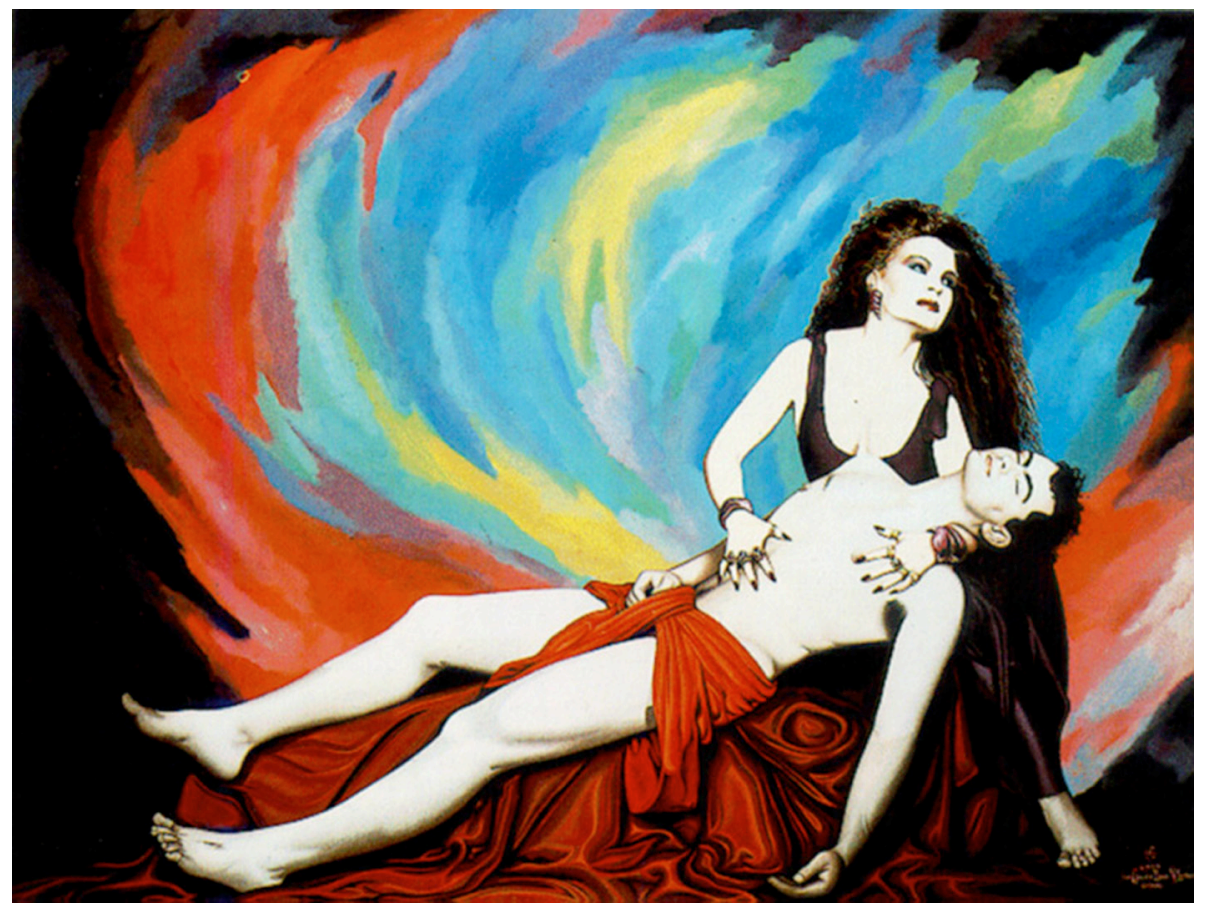

3. Las Costus, Patria (de la serie El Valle de los Caídos), 1986

underground español y el movimiento contracultural barceIonés, conocido como El Rrollo, tendrá un gran desarrollo, y del que formarán parte tanto artistas pertenecientes a la ciudad como procedentes de otras regiones del país, como es el caso de los sevillanos Nazario y José Pérez Ocaña [2] ${ }^{17}$ No obstante, a finales de los setenta, el surgimiento de la movida madrileña acaparó el protagonismo mediático siendo especialmente importante para los movimientos homosexuales por su ideología, en la que las etiquetas como homo o heterosexual eran vistas como el resultado de un discurso autoritario por lo que abogaban por la ambigüedad ${ }^{18}$.

La producción artística que se generó en ambas ciudades estuvo dominada por un discurso en el que se sexualizó el cuerpo, tal y como se puede comprobar tanto en la obra de Nazario como en algunas de las acciones callejeras de Ocaña en Barcelona ${ }^{19}$. Estas acciones, asimismo, ejemplifican otra de las cualidades de los discursos generados a partir de los movimientos contraculturales, la subversión y parodia que se hizo de los valores del nacional-catolicismo destacando, por su parte, dentro del contexto madrileño a Las Costus ${ }^{20}$. Interesados en homenajear a Madrid, produjeron su serie El Valle de los Caídos [3] en la que emplearon a personalidades de la movida, por ejemplo a Alaska, como modelos para imitar el programa escultórico del monumento franquista ${ }^{21}$.

Asimismo pueden localizarse otros artistas que también en los setenta comienzan a trabajar sobre la heteronormatividad y la indefinición en relación al género y el sexo. El principal ejemplo sería el artista canario Juan Hidalgo por obras como Hombre, mujer y mano o Biozaj apolíneo Biozaj dionisiaco [4] (ambas de 1977) en las que entremezcla la geografía corporal del hombre y la mujer llegando a representar cuerpos a medio camino entre ambos. Asimismo merece la pena destacar también de esta misma década las figuras encapsuladas [5] de Darío Villalba que abarca esta problemática desde una perspectiva más amplia. Si bien en las primeras empleó el color, progresivamente este fue desapareciendo. Estas series consisten en fotografías de gran formato dentro de pompas o cápsulas de metacrilato. Tanto los retratos distantes y cargados de dramatismo como el hecho de que se encuentren encerrados nos hablan de la imposición de una serie de dictámenes que marcan tanto la vida pública como la privada creando con ello un contexto de profunda opresión en todos los ámbitos.

Dentro de este ámbito en el que el activismo LGTB da sus primeros pasos, además de surgir los primeros textos 


\section{Juan Hidalgo, Biozaj apolíneo} Biozaj dionisíaco, 1977

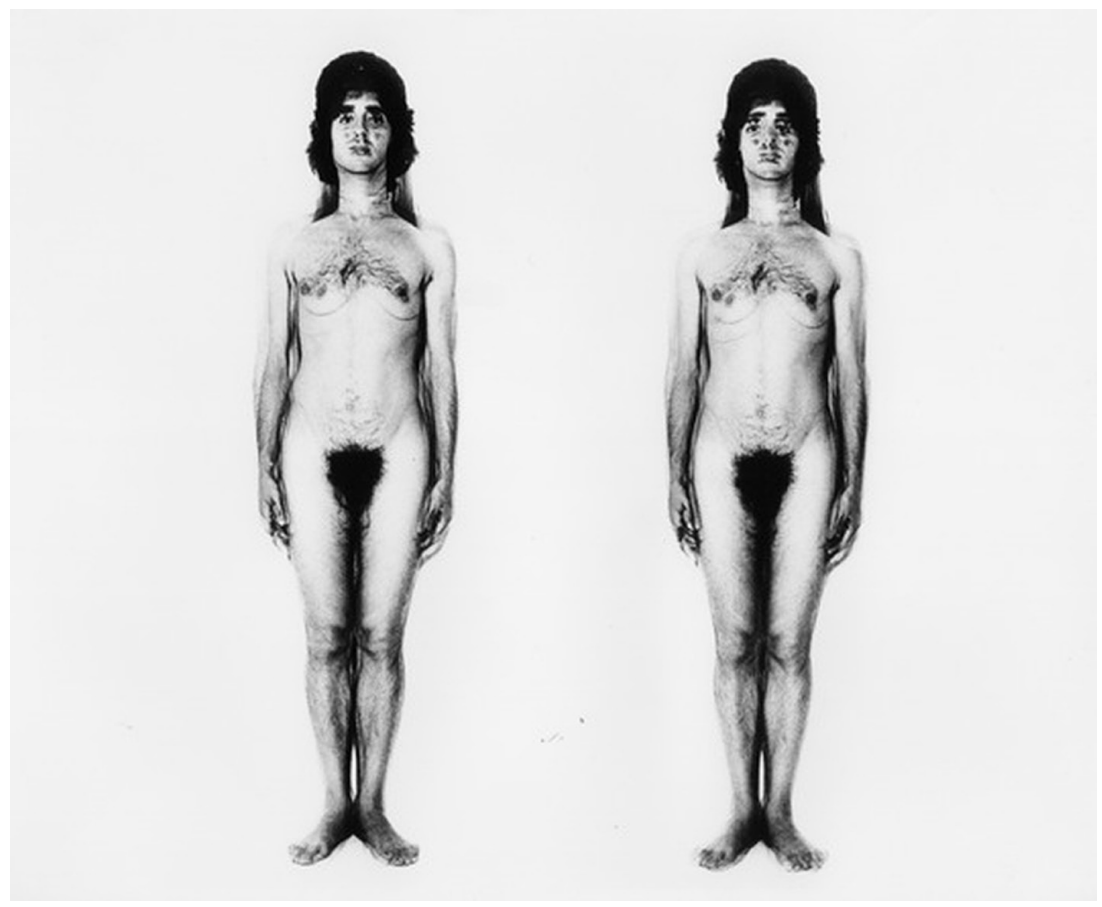

sobre homosexualidad en clave reivindicativa, se generan las primeras publicaciones dirigidas al público homosexual como Party (1976)22. Pese a ello, los años setenta y ochenta brillan por la escasez de teóricos interesados en la cuestión gay. Paul Julian Smith explica esta situación al comprobar que, en aquel momento, cualquier persona que hubiera estado interesada por este tipo de temas no habría encontrado cabida dentro del sistema académico-cultural español ${ }^{23}$.
Esto provocó la emigración de una parte de la población estudiantil, investigadora y activista hacia Estados Unidos, Inglaterra y Francia, donde entró en contacto con los discursos de género internacionales. En esta situación, destaca la figura del antropólogo Alberto Cardín como uno de los pocos referentes intelectuales del mundo LGTB de los $80^{24}$. Su obra, conjunta con Armand de Fluvià, Sida ¿maldición bíblica o enfermedad letal?, puso de manifiesto la pobreza
5. Retrospectiva de Darío Villalba en el MNCARS, 2007

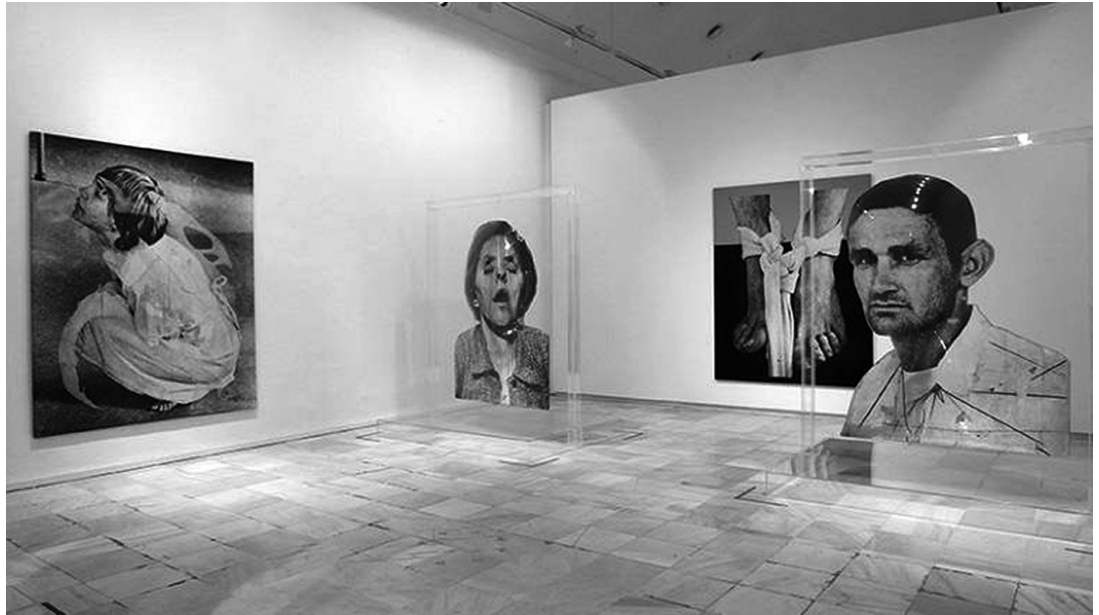




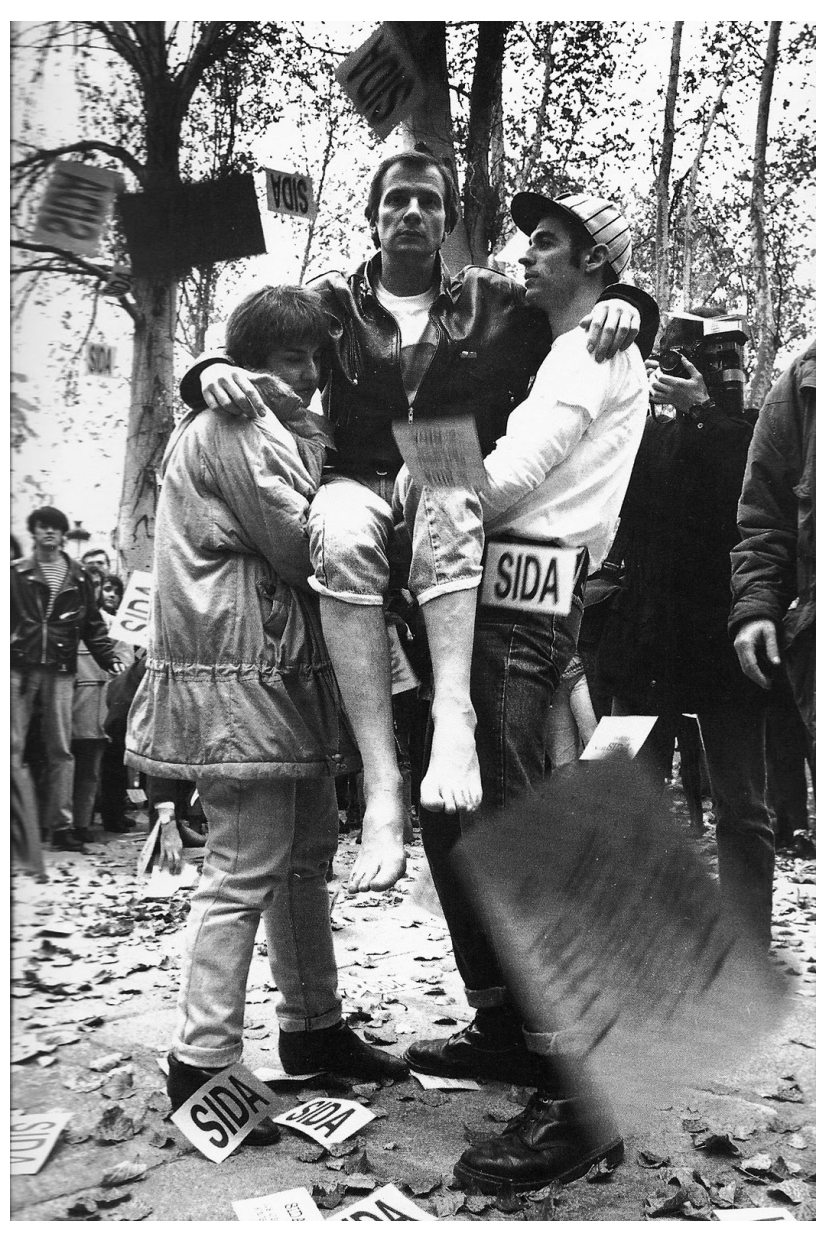

6. Pepe Espaliú, Acción carrying, San Sebastián, 1991

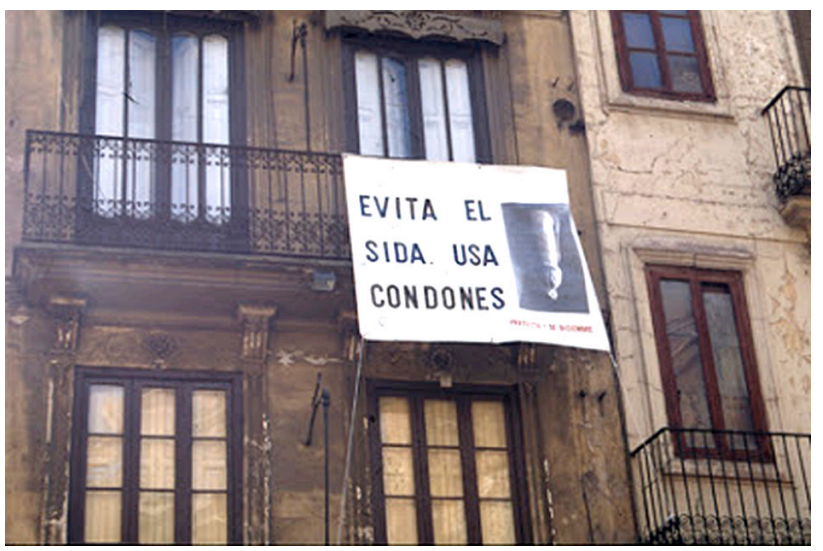

7. Proyecto 1 de diciembre, intervención en el espacio público, Valencia, 1992 científica y conceptual de la producción teórica española con respecto a esta enfermedad ${ }^{25}$.

Con la llegada de los noventa se produce un punto de inflexión debido a una intensificación de la influencia internacional. Ya en los últimos años del franquismo el movimiento gay se hizo eco de algunos de los debates teóricos del ámbito académico extranjero. No obstante, a finales de los ochenta y en los noventa se produce el retorno de aquellos que habían emigrado al extranjero y habían accedido a una serie de materiales bibliográficos y formado parte de un activismo del que España carecía ${ }^{26}$.

Gracias a ello y precedidos en 1991 por ACT UP Barcelona, se fundarán en 1993 y 1994, bajo la estela del activismo antisida estadounidense, LSD y La Radical Gai. Ambos emplearon por primera vez en el territorio nacional el término queer e intentaron difundirlo a través de sus publicaciones, Non Grata y De un Plumazo, respectivamente. Además de esto fueron los grupos cohesionados más importantes que llevaron a cabo un frente de lucha abierto contra la respuesta del Estado ante la enfermedad del SIDA. Además comienza a desarrollarse en los inicios de la década una corriente artística y teórica en la que esta enfermedad se convierte en objeto de reflexión. Así, si bien el número de artistas que formaron parte de esta línea de investigación fue muy reducido, cabría destacar la personalidad de Pepe Espaliú, quien formó parte del activismo neoyorquino y, a raíz de esta experiencia, concibió su acción carrying [6] en $1992^{27}$. Igualmente, fue Espaliú el que puso en contacto con el grupo activista estadounidense ACT UP a Juan Vicente Aliaga $^{28}$. Este publicó un año más tarde, junto con José Miguel García Cortés, De amor y Rabia, que, junto con Construyendo sidentidades, pasó a ser un libro clave de la producción teórica sobre el sida en España ${ }^{29}$. Progresivamente se generó un tejido de trabajo donde se produjeron distintas colaboraciones entre artistas, profesores, estudiantes y teóricos siendo el Proyecto 1 de diciembre, una asociación formada por alumnos y profesores de la facultad de Bellas Artes de la Universidad Politécnica de Valencia, el resultado más importante de este proceso [7].

Asimismo, a lo largo de la década de los noventa comenzaron a traducirse al castellano algunos textos esenciales para los estudios de género, como los de Judith Butler, Donna Haraway, Guy Hocquenghem y Eve Kosofsky Sedwick lo que, a su vez, permitió difundir las teorías feministas 
y queer con las que se estaba trabajando en el resto de Europa y en Estados Unidos ${ }^{30}$. Sin embargo, si la introducción de los discursos feministas internacionales en el panorama español permitió a la academia actualizarse en este campo de estudio, por otra parte conllevó que este sector prefiriese no investigar su propio pasado que era, en comparación, menos atractivo ${ }^{31}$. Esto provocó que el primer grupo de artistas y teóricas feministas dentro del Estado español, denominado por Navarrete, Ruido y Vila como «generación de los noventa», careciera de referentes artísticos españoles, nutriéndose, sobre todo, de los contextos anglosajón y estadounidense ${ }^{32}$. Con la progresiva revisión historiográfica que se irá desarrollando a lo largo del nuevo siglo, este problema se evidenció en proyectos como Desacuerdos pero, sobre todo, en la exposición Genealogías Feministas en el arte español (1960-210) (MUSAC, 2012).

No correrá la misma suerte la introducción de la teoría queer. Su importación al ámbito académico en un intento por actualizar el panorama cultural español fue llevada a cabo por académicos que, como Juan Vicente Aliaga, que trabajó como profesor en la Oxford University entre 1985 y 1987, habían mantenido contacto con el mundo académico y activista inglés, francés o estadounidense. El hecho de que España no hubiera contado con un activismo y una línea teórica y de trabajo feminista que generara unos planteamientos iniciales a partir de los que abrir un debate que cuestionara el discurso heteronormativo en torno a la sexualidad hizo que, inicialmente, esta teoría no tuviera gran acogida.

Pese a ello, en el ámbito artístico ya en los noventa se pueden localizar un número cada vez más creciente de artistas interesados por las problemáticas de sexo y género y que, de hecho, fueron recogidos en la exposición Transgenéric@s: representaciones y experiencias sobre la sociedad, la sexualidad y los géneros en el arte español contemporáneo (Koldo Mitxelena Kulturunea, 1998) ${ }^{33}$. El referente de mayor importancia para la concepción de esta exposición fue In a Different Light. Visual culture, sexual identity, queer practice (University of California, Berkeley, 1995) puesto que ambas trabajaron dentro de unas líneas conceptuales muy similares. Sin embargo, la exposición estadounidense abarcaba un arco cronológico y geográfico mucho más amplio, el arte norteamericano del siglo XX, y se centraba en la experiencia gay/lésbica, mientras que en Transgenéric@s, a pesar de que también la homosexualidad aparecía como tema

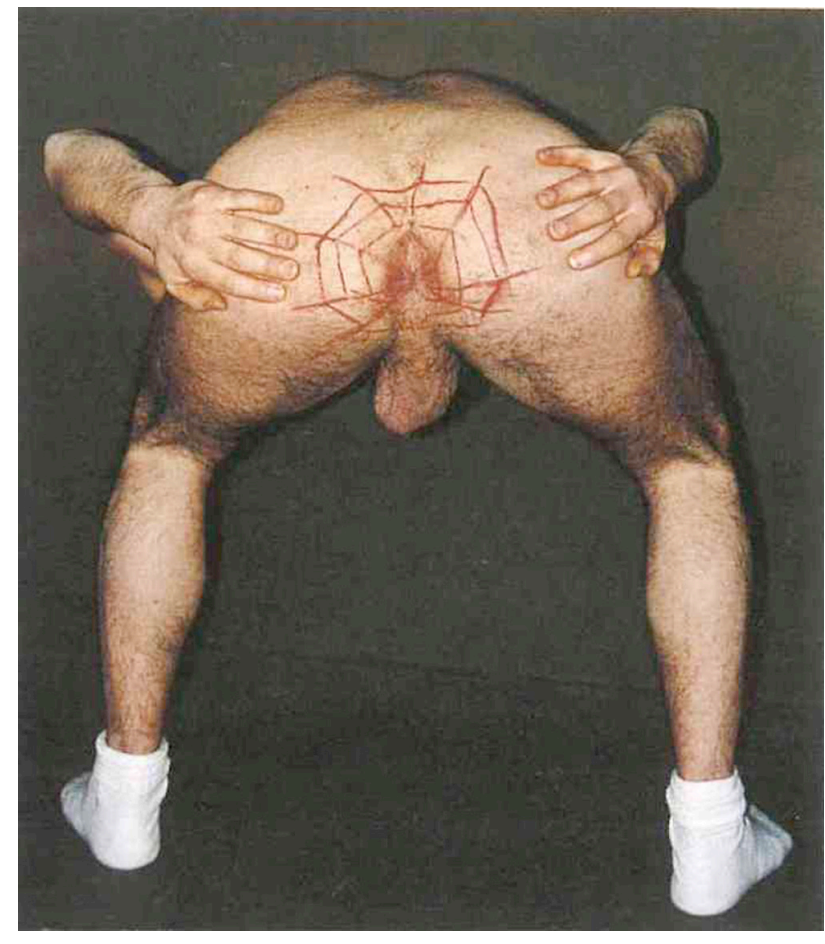

8. Jesús Martínez Oliva, Sin título (Serie Miedos y Fobias), 1995-1998

de alguna obra, fue pensada como una exposición en la que se deconstruyeran los binarismos imperantes relacionados con la sexualidad: hombre/mujer, masculinidad/femineidad, activo/pasivo, activa/pasiva, etc.

El planteamiento de la exposición, más que transmitir un mensaje claro al espectador, consistió en mostrar un compendio de artistas pertenecientes a un mismo contexto, la España de los noventa, que trabajaban dentro de un mismo campo de acción, la deconstrucción de las identidades estables, pero a través de distintas líneas de investigación. Así, Jesús Martínez Oliva habla de los límites de la construcción de la masculinidad dentro de la sociedad heteronormativa partiendo de la abyección y de lo anal [8]; Alex Francés, por su parte, juega con la idea de la pasividad y la connotación negativa que la sociedad tiene de ella en relación al hombre; Cabello y Carceller abordaron la legitimidad del amor lesbiano [9], etc. Asimismo también supuso la institucionalización del colectivo LSD que expuso obras que habían formado parte de las exposiciones Es-Cultura Lesbiana y Menstruosidades. 


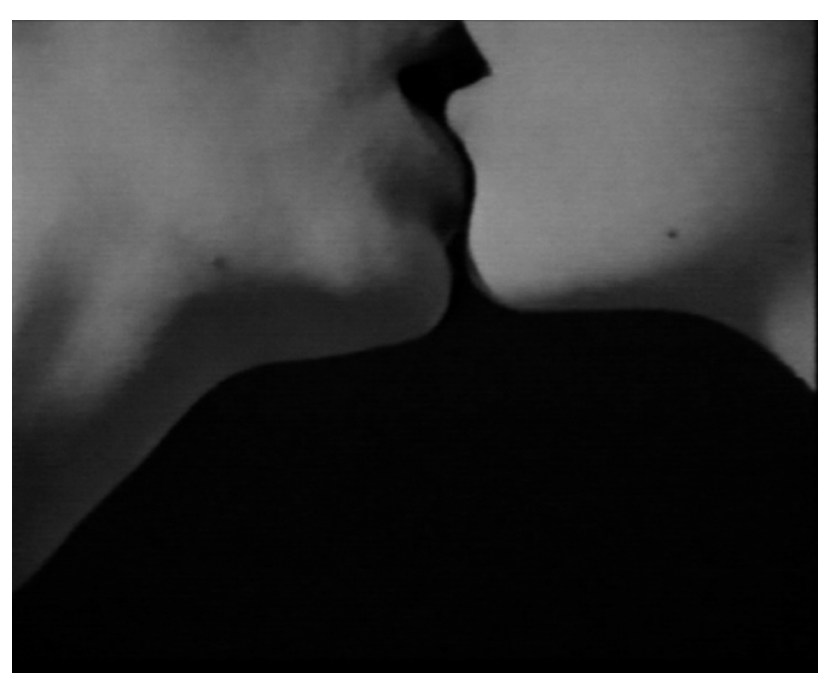

9. Helena Cabello y Ana Carceller, El beso, 1996

Otro de los problemas que ha presentado su recepción en España es el hecho de que la palabra inglesa queer no posea una traducción específica al español, cuestión que ha sido objeto de debate entre los investigadores de nuestro país. En los noventa La Radical Gai intentó con escaso éxito establecer el término "transmaricabollo» como equivalente al concepto inglés. Paralelamente, ha habido una clara oposición por parte de autores como Javier Sáez, quien ha reivindicado el valor contradiscursivo del término inglés queer y ha criticado tanto el intento de traducirlo como el uso genérico que algunos como Herrero Brassas han hecho de él, desactivándolo ${ }^{34}$. Otros como Beatriz Preciado sitúan esa necesidad por traducir el término queer en que es visto como un producto de la cultura norteamericana, lo que imposibilita su uso en otros contextos. Para ella, sin embargo, el concepto surge como una crítica no al discurso heteronormativo estadounidense, sino occidental, lo que legitima su uso en España ${ }^{35}$.

Entre otras, una de las traducciones que recibirá será la de "teoría torcida» que, de hecho, será el título del primer ensayo queer español en el que su autor, Ricardo Llamas, explica los entresijos de dicha teoría ${ }^{36}$. Esta publicación, junto con la inauguración en 1997 de la exposición El Rostro Velado: travestismo e identidad en el arte y en 1998 de Transgenéric@s marcará la entrada definitiva de la teoría queer en el país. A partir de este momento, esta teoría, precedida por los escritos pertenecientes a los noventa, se desarrolló en un número creciente de publicaciones, destacando especialmente Manifiesto contrasexual de Beatriz Preciado ${ }^{37}$.

En el ámbito de las instituciones culturales fue a partir de 2003 cuando comienzan a organizarse un número cada vez mayor de actividades, no solo referentes al feminismo, sino también a la teoría queer y a la teoría de las masculinidades. Hay que destacar especialmente el seminario Introducción a la teoría queer organizado por David Córdoba, Javier Sáez y Francisco Vidarte en el marco de la UNED ${ }^{38}$. En él se creó un espacio de reflexión, ya no solo sobre el nacimiento de la teoría queer desde distintas perspectivas, sino también del modo en el que se había ido introduciendo en España. La importancia de este seminario se localiza en el hecho de que, por primera vez en el panorama nacional, una universidad apoyaba abiertamente un programa cultural enfocado hacia el queer consiguiendo un mayor alcance y difusión.

Asimismo, la postpornografía, consistente en una revisión de los códigos iconográficos y de los estereotipos de sexo y género establecidos en la industria pornográfica comercial, ganará un gran protagonismo gracias a la Maratón Posporno organizada por Beatriz Preciado en el MACBA en $2003^{39}$. Esta consistió en un seminario-taller de dos días en los que se abordó la estética y el concepto de postpornografía y se creó un espacio de debate en torno a las relaciones entre la pornografía, la arquitectura, el feminismo y el sadomasoquismo. A pesar de que algunos años antes otras instituciones como Arteleku y el propio MACBA habían acogido actividades relacionadas con el feminismo, la Maratón fue una de las primeras ocasiones en las que un colectivo LGTB colaboraba con una institución cultural ${ }^{40}$. Además, a raíz de ella se gestaron grupos como Post_Op (2003), eventos como la Muestra Marrana (2004) y seminarios y talleres en distintas instituciones repartidas por el espacio geográfico español como Arteleku o el espacio Off Limits ${ }^{41}$.

Cabe destacar el papel de Beatriz Preciado, impulsora de un gran número de programas sobre los discursos postidentitarios en distintos centros culturales, artísticos y museísticos. Ha tenido especial relevancia, sobre todo, por sus clases y talleres dentro de proyectos como UNIA arteypensamiento y en el Programa de Estudios Independientes (PEI) del MACBA originado a raíz del éxito del seminario Tecnologías del Género dirigido por ella. Preciado, debido al hecho 
de haber investigado junto a Jacques Derrida, y al éxito que cosechó con el Manifiesto contrasexual42, consiguió sortear con mayor facilidad las barreras que se imponen para acceder a las distintas instituciones en las que ha trabajado y pasar a ser una de las principales impulsoras de estas actividades en España ${ }^{43}$. De hecho, en 2012 participó en la creación de Somateca en el MNCARS, espacio donde se «exploran las prácticas políticas y artísticas de producción, normalización y crítica del cuerpo que surge en el contexto de la modernidad, desde la ilustración hasta la aparición del SIDA pasando por las mutaciones del liberalismo posteriores a la Segunda Guerra Mundial» ${ }^{44}$. Somateca ha llegado a tener tal acogida y éxito que la tercera edición, llevada a cabo en 2014, debido a la falta de fondos para el programa por parte del museo, ha sido constituida por estudiantes de las dos ediciones anteriores.

Al igual que las asociaciones feministas han producido durante los últimos años festivales como Feministaldia (creado en 2006) o Miradas de mujer (creado en 2012), lo mismo ha sucedido en relación al colectivo LGTB y al queer a lo largo de la última década. El primero de ellos fue el Festival Visible, comisariado por el gestor cultural Pablo Peinado en 2005, como un complemento cultural y activista a las fiestas del Orgullo Gai de Madrid. A pesar de ser uno de los festivales culturales LGTB más importantes del mundo, debido a la crisis económica, irá perdiendo financiación para, finalmente, en 2012, organizar su última edición. Con todo, hay que destacar que la importancia que tuvo este festival conllevará en 2007, gracias a una serie de amigos activistas gallegos, la organización A Coruña Visible, un festival paralelo al madrileño ${ }^{45}$. Un objetivo similar es el que ha perseguido otro de los festivales, Muestra-T. Nacido en 2006 al amparo de la Federación Estatal de Lesbianas, Gais, Transexuales y Bisexuales (FELGTB), la diferencia principal que mantiene con respecto a los dos anteriores sería el hecho de que este nació, y hasta la fecha lo sigue siéndolo, como un festival puramente institucionalizado derivado de las fiestas del Orgullo Gay organizadas por el Ayuntamiento de Madrid. Por último, el caso más reciente ha sido el de Queer Mad, cuya dirección artística fue ocupada por el performer navarro Abel Azcona. Formado por talleres, ciclos de cine, performances y representaciones teatrales entre otros eventos, ha conseguido crear un tejido colaborativo entre distintos agentes procedentes de diversos ámbitos ${ }^{46}$.
Al igual que las «exposiciones de mujeres» de los noventa el principal aspecto positivo de todos estos festivales y ciclos es el de haber conseguido dar una mayor visibilidad a un cierto número de artistas y propuestas de sumo interés que, de otro modo, seguirían en un segundo plano. No obstante, han supuesto una cierta mercantilización de la producción artística relacionada con los estudios de género haciendo que, en algunos casos, arte feminista vuelva a ser equiparado con el arte producido por mujeres o que el arte queer lo sea con el arte gay o marica ${ }^{47}$.

Echando la vista atrás tal y como se ha hecho a lo largo de este artículo, una de las principales conclusiones que pueden extraerse es el hecho de que pese al gran desarrollo que la teoría queer ha tenido en nuestro país, hay que tener en cuenta una diferencia fundamental con respecto a Estados Unidos y Reino Unido. Mientras que en sendos países, queer fue empleado en su vertiente subversiva en el activismo de la calle para, posteriormente, ser teorizada en los círculos académicos, en España dicho proceso se produjo a la inversa. Fue un concepto importado por parte de unos pocos y en torno al que trabajaron grupos como LSD y La Radical Gai quienes, faltos de una formación teórica sólida, no terminaron de entender el concepto en su totalidad. Esto se demuestra al comprobar cómo LSD mostró en sus inicios una gran aversión a la masculinidad femenina, algo que la teoría queer defiende y justifica ${ }^{48}$.

No obstante, la conclusión más importante y que, de hecho, es la que ha motivado la producción de este texto, es que, pese al impulso de revisión historiográfica iniciado en España hace más de una década, no se ha reflexionado desde un punto de vista crítico en torno a la aplicación y el desarrollo de la teoría queer.

De este modo, en primer lugar, si observamos quiénes han desarrollado la mayor parte de las exposiciones y actividades en instituciones públicas podremos comprobar como casi siempre se repiten los mismos nombres: Juan Vicente Aliaga, José Miguel García Cortés y Beatriz Preciado, que han generado un discurso hegemónico en torno a los estudios de género y la teoría queer.

En segundo lugar tampoco se ha reflexionado sobre el carácter hegemónico del término queer. La necesidad de ello no viene motivada por el cuestionamiento del campo de acción del discurso puesto que, como bien explica Beatriz Preciado, es un discurso extensible al contexto euroame- 
ricano, sino por el concepto en sí mismo. Hablar de arte queer español resulta, desde la perspectiva generalizada según la que el concepto es traducido por «raro» (amén de otras traducciones), inexacto puesto que si en inglés conlleva una carga peyorativa o subversiva, en España, al encontrarnos en un contexto social en el que la lengua es diferente y en el que, por lo tanto, queer no significa nada, el concepto es liberado de dichas connotaciones. Esto provocaría que el actual intento de establecer una genealogía queer española partiendo de Ocaña resulte fallido desde un punto de vista de rigor conceptual, no solo por emplear de un modo anacrónico el término, sino porque para poder considerarlo como tal habría que analizar solo sus acciones transformis- tas dejando de lado su obra pictórica, dando, por tanto, una visión sesgada tanto de él como de su obra.

No obstante, la otra perspectiva desde la que puede abordarse esta problemática conceptual consiste en aprovechar que el término queer no tiene traducción y que en español resulta imposible poder asociarlo a un género o sexo. Así, si vaciamos el concepto de todas las connotaciones culturales pertenecientes al mundo anglosajón y nos apropiamos de él, podríamos responder que sí, que existe un arte queer español actualmente. Un tipo de arte que, al igual que queer ${ }^{49}$, sea inclasificable en un sistema basado en binomios lingüísticos y culturales y que, a la vez, destruya los roles de género, tanto los tradicionales como los más contemporáneos.

\section{Notas}

1 ALIAGA, Juan Vicente, «¿Existe un arte queer en España?», en: http://www.accpar.org/numero3/queer.htm (Fecha de consulta: 28-08-2014).

2 PRECIADO, Beatriz, Manifiesto contra-sexual, Madrid, Opera Prima, 2002, p. 24.

3 MIRA, Alberto, De Sodoma a Chueca. Una historia cultural de la homosexualidad en España en el siglo XX, Madrid, Egales, 2004, p. 297.

4 Ley de Vagos y Maleantes de 1933, disponible en: http://galeon.com/franquismo/web/LVaMa.pdf (Fecha de consulta: 28-08-2014).

5 MIRA, De Sodoma a Chueca..., p. 321.

6 Ley de Peligrosidad y Rehabilitación Social, disponible en: https:/www.boe.es/boe/dias/1970/08/06/pdfs/A12551-12557.pdf (Fecha de consulta: 28-082014).

7 PRECIADO, Beatriz, "Campceptualismos del Sur. Ocaña y la historiografía española». En: http://www.macba.cat/es/seminario-tropicamp (Fecha de consulta: 13-04-2014).

8 Euskal Herriko Gay Askapen Mugimendua en el País Vasco (1976), Moviment d'Alliberament Sexual del País Valencià (1977) y el Frente Homosexual de Acción Revolucionaria en Madrid (1977).

9 MARZO, Jorge Luis, «Arte, conflicto y poder». En: http://www.soymenos.net/Arte,\%20conflicto\%20y\%20poder.pdf (Fecha de consulta: 26-02-2014). MARTínEZ, Guillem (ed.), CT o la Cultura de la Transición. Crítica a 35 años de cultura española, Barcelona, Debolsillo, 2012.

10 MAYAYO, Patricia, "Imaginando nuevas genealogías. Una mirada feminista a la historiografía del arte español contemporáneo", en ALIAGA, Juan Vicente; MAYAYO, Patricia (eds.), Genealogías feministas en el arte español (1960-2010), Madrid, This Side Up, 2013, pp. 33-34.

11 ALIAGA, « ¿Existe un arte queer en España?».

12 MIRA, De Sodoma a Chueca..., p. 476.

13 La primera agrupación homófila fue Agrupación Homófila para la Igualdad Sexual (AGHOIS) fundada en 1970 que, dos años más tarde, pasará a ser el Movimiento Español de Liberación Homosexual (MELH).

14 Nazario y amigos charlan sobre la Barcelona de los 70. En: https://www.youtube.com/watch?v=Jjg_p0Pkb20 (Fecha de consulta: 18-05-2014).

15 En Estados Unidos la primera asociación homófila, la Sociedad Mattachine, se fundó en 1950 en Los Ángeles mientras que el primer grupo activista se formó en 1969 en Nueva York con el nombre de Gay Liberation Front.

16 RIZZO, Domenico, «Esfera pública y políticas gays desde la Segunda Guerra Mundial», en ALDRICH, Robert (ed.), Gais y lesbianas. Vida y cultura. Un legado universal, Madrid, Editorial Nerea, 2005, p. 214.

17 MIRA, De Sodoma a Chueca..., pp. 544-545.

18 Ibid., pp. 517-520.

19 Esto se puede comprobar en cómics de Nazario pertenecientes a la primera mitad de los setenta como El rollo enmascarado (1973) o Piraña divina (1975). PRECIADO, Beatriz, «La Ocaña que merecemos. Campceptualismo, subalternidad y políticas performativas», en GONZÁLEZ ROMERO, Pedro (com.), Ocaña. 1973-1983: acciones, actuaciones, activismo, Barcelona-San Sebastián, La Virreina-Centro Cultural Montehermoso Kulturenea Vitoria-Gasteiz, 2011, p. 90.

20 Pareja formada por el gaditano Enrique Naya y el mallorquín Juan José Carrero, quienes en 1981 decidieron constituirse como Las Costus, sobrenombre procedente de «costureras» debido al gran número de horas que pasaban junto a una mesa camilla.

21 La serie de El Valle de los Caídos fue iniciada en 1979 pero la exposición en Casa de Vacas no tuvo lugar hasta 1987. SANZ CASTAÑO, Héctor, «Institucionalización y marginalidad del arte desviado en la Transición española», en RAMíREZ, Juan Antonio (ed.), El sistema del arte en España, Madrid, Ediciones Cátedra, 2010, pp. 355-356.

22 Otras fueron: ANABITARTE, Héctor, Homosexualidad: el asunto está caliente, Madrid, Quiemada, 1979 y FLUVIÁ, Armand, Aspectos jurídico-legales de la homosexualidad, Barcelona, Institut Lambda, 1979.

23 MIRA, De Sodoma a Chueca..., p. 466.

24 VÉLEZ-PELLEGRINI, Laurentino, Sujetos de un contra-discurso. Una historia intelectual de la producción teórica gay, lesbiana y queer en España, Barcelona, Ediciones Bellaterra, 2011, p. 37.

25 CARDÍN, Alberto y FLUVIÀ, Armand, Sida ¿Maldición bíblica o enfermedad letal?, Barcelona, Laertes, 1985. 
GARCÍA CORTÉS, José Miguel, «A Alberto Cardín in memoriam» en ALIAGA, Juan Vicente y GARCÍA CORTÉS, José Miguel, De amor y rabia. Acerca del arte y el sida, Valencia, Universidad Politécnica de Valencia, 1993, pp. 168-169.

26 Entrevista a las artistas Helena Cabello y Ana Carceller el 27/06/2014. BUXÁN BRAN, Xosé Manuel, Radicales libres. Experiencias gays y lesbianas en el arte peninsular, La Coruña, Auditorio de Galicia, 2005, p. 328.

27 Entre los artistas interesados en esta línea de trabajo estuvieron Pepe Espaliú, Pepe Miralles, Jesús Martínez Oliva, Javier Codesal, Roberto González Fernández, Alejandra Orejas, Sonia Guisado y Mu-ur Vindel. En el caso de Pepe Espaliú, su obra más conocida fue el carrying. La palabra proviene del intercambio que a veces se hacía de la palabra carrying (llevar) por caring (cuidar) en las asociaciones de apoyo al sida de Nueva York, donde un grupo de cuidadores, los buddies, ayudaban a los enfermos en sus quehaceres diarios. La idea del carrying tuvo dos modalidades: por un lado, su materialización como palanquín, que simbolizaba ese distanciamiento que mantenía la gente con los seropositivos, y, por otro, como acción, que consistía en llevarlo en volandas por parejas a lo largo de un recorrido determinado. En este segundo caso se llevó a cabo en dos ocasiones, la primera de ellas en San Sebastián y la segunda en Madrid. Para más información: ALIAGA, Juan Vicente, «Terreno de lucha. El impacto de la sexualidad y la huella del sida en algunas prácticas artísticas performativas». En: http://www.macba.cat/uploads/20140211/QP_14_Aliaga.pdf (Fecha de consulta: 29-08-2014).

28 VÉLEZ-PELLEGRINI, Sujetos de un contra-discurso..., p. 89.

29 LLAMAS, Ricardo, Construyendo identidades: estudios desde el corazón de una pandemia, Madrid, Siglo XXI, 1995.

30 BUTLER, Judith, El género en disputa: el feminismo y la subversión de la identidad, Barcelona, Paidós, 2007; HARAWAY, Donna, Ciencia, cyborgs y mujeres: la reinvención de la naturaleza, Madrid, Cátedra, 1995; HOCQUENGHEM, Guy, El deseo homosexual, Barcelona, Melusina, 2009; KOSOFSKY SEDGWICK, Eve, Epistemología del armario, Barcelona, Ediciones de la Tempestad, 1998.

31 MAYAYO, «Imaginando nuevas genealogías...», p. 35.

32 NAVARRETE, Carmen; RUIDO, María; VILA, Fefa, «Trastornos para devenir: entre artes y políticas feministas y queer en el Estado español», en AA. W., Desacuerdos. Sobre arte, políticas y esfera pública en el arte español n. ${ }^{2}$. Barcelona, Sevilla, San Sebastián: Museo d'Art Contemporani de Barcelona, Universidad internacional de Andalucía-arteypensamiento, Arteleku, 2005, pp. 158-187 op. cit., p. 171. MAYAYO, «Imaginando nuevas genealogías...», p. 32.

33 Los artistas que participaron en esta exposición fueron Juan Pablo Ballester, Miguel Benlloch, Nuria Canal, Cabello y Carceller, Carles Congost, Ricardo Cotanda, Txaro Fontalba, Alex Francés, Nuria León, LSD, Jesús Martínez Oliva, Chelo Matesanz, Joan Morey, Carmen Navarrete, Marina Núñez, Estibalitz Sabada, Eduardo Sourrouille, Eulèlia Valdosera y Azucena Vieites.

34 VÉLEZ-PELLEGRINI, Sujetos de un contra-discurso..., p. 72.

35 CARRILLO, Jesús, «Entrevista a Beatriz Preciado», en AA. W., Desacuerdos. Sobre arte, políticas y esfera pública en el arte español, n. 2 , Barcelona, SeviIla, San Sebastián, Museo d'Art Contemporani de Barcelona, Universidad Internacional de Andalucía-arteypensamiento, Arteleku, 2005 , p. 251.

36 Teoría marica, teoría rosa, teoría trasgresora, etc. LLAMAS, Ricardo, Teoría torcida, Madrid, Siglo XXI, 1998.

37 GUASCH, Óscar, La sociedad rosa, 1995; BUXÁN BRAN, Xosé Manuel, Conciencia de un singular deseo. Estudios lesbianos y gays en el Estado Español, 1997; ALIAGA, Juan Vicente, Bajo vientre. Representaciones de la sexualidad en la cultura y arte contemporáneo, 1997; ALIAGA, Juan Vicente, GARCÍA CORTÉS, José Miguel, Identidad y diferencia, 1997; VIDARTE, Francisco; LLAMAS, Ricardo, Homografías y Extravíos, 1999; VIÑUALES, Olga, Identidades lésbica, 2000; PRECIADO, Beatriz, Manifiesto contrasexual, 2002; MIRA, Alberto, Para entendernos. Diccionario de cultura homosexual, gay y lésbica, 2002; GUASCH, Ó., VIÑUALES, O., Sexualidades. Diversidad, 2003; ALIAGA, Juan Vicente, Arte y cuestiones de género, 2004; MIRA, De Sodoma a Chueca...; GARCÍA CORTÉS, José Miguel, Hombres de mármol. Códigos de representación y estrategias de poder de la masculinidad, 2004; SÁEZ, Javier, Teoría queer y psicoanálisis, 2004; MARTíNEZ OLIVA, Jesús, El desaliento del guerrero: Representaciones de la masculinidad en el arte de las décadas de los 80 y 90, 2005; PRECIADO, Beatriz, Testo Yonki, 2008; VÉLEZ-PELLEGRINI, Sujetos de un contra-discurso..., etc.

38 El seminario se realizó entre 2003 y 2005 y en él participaron David Córdoba, Beatriz Preciado, Sejo Carrascosa, Carmen Romero Bachiller, Alfonso Ceballos Muños, Desiré Rodrigo, Helena Rodríguez, Marcelo Soto, Eduardo Nabal, Pablo Pérez Navarro y Fefa Vila.

39 En ella participaron Beatriz Preciado, Juan Antonio Suárez, Javier Sáez, Joan Pujol, Helena Torres, Marie-Hélène Bourcier, Fefa Vila y el dúo LICIT, formado por Diana Zapata y Margarita Carreras.

40 TRAFÍ-PRATS, Laura, "De la cultura feminista en la institución del arte», en AA. W., Desacuerdos. Sobre arte, políticas y esfera pública en el arte español n. ${ }^{\circ}$ 7. Barcelona, Sevilla, San Sebastián, Granada, Madrid, Museo d'Art Contemporani de Barcelona, Universidad internacional de Andalucía-arteypensamiento, Arteleku, Centro José Guerrero, Museo Nacional Centro de Arte Reina Sofía, 2012, p. 215.

41 Tras su primera edición la Muestra Marrana, se han organizado una anualmente en Barcelona y a partir de 2009 dos anuales, una en Barcelona y otra en Madrid.

Para ver las actividades referidas al feminismo, la postpornografía y la teoría queer ver Anexo 3

42 VÉLEZ-PELLEGRINI, Laurentino, «Teoría queer: de la esperanza al gran fraude», El viejo topo, n. ${ }^{\circ} 281,2011$, p. 47.

43 Ver Anexo 1.

44 «Folleto del programa de Somateca 2013». En: http://www.museoreinasofia.es/actividades/somateca-2013-vivir-resistir-condicion-neoliberal (Fecha de consulta: 10-03-2014).

45 Entrevista al gestor cultural Pablo Peinado Céspedes el 09/05/2014.

46 Espacios culturales como Factoría de Arte y Desarrollo, espacios expositivos como Espacio Trapezio y Serendipia, espacios lúdicos como Fulanita, etc.

47 Entiéndase en este caso gay en el sentido inglés referido tanto a hombres como a mujeres homosexuales.

48 Entrevista a las artistas Helena Cabello y Ana Carceller el 27/06/2014.

49 Hay que tener en cuenta que, al descontextualizar la palabra y al apropiarse de ella deja de ser un término extranjero por lo que no son necesarias las cursivas. 
